\title{
In vitro anti-diabetic activity, bioactive constituents, and molecular modeling studies with sulfonylurea receptor 1 for insulin secretagogue activity of seed extract of Syzygium cumini (L.)
}

\author{
Meharban Asanaliyar ${ }^{\circledR}$, Pratibha Nadig ${ }^{1 *}$, Nagakumar Bharatam ${ }^{2}{ }^{\circledR}$ \\ ${ }^{1}$ Department of Pharmacology, Vydehi Institute of Medical Sciences and Research Centre, Bangalore 560066, Karnataka, India \\ ${ }^{2}$ Bugworks research India Pvt.Ltd, Center for Cellular \& Molecular Platforms, National Center for Biological Sciences, TIFR GKVK Campus, Bellary \\ Road, Bangalore 560 065, India
}

\section{A R T I C L E I N F O}

\section{Article Type:}

Original Article

\section{Article History:}

Received: 4 December 2020

Accepted: 5 February 2021

\section{Keywords:}

Molecular docking

Spectroscopy

Gallic acid

Ellagic acid

RIN-5F cells

L6 myoblasts

\begin{abstract}
A B S T R A C T
Introduction: Syzygium cumini (L.) has been known to be used for diabetes treatment in traditional Indian and Chinese medicine. The present study focuses on the evaluation for glucose uptake and insulin release in vitro and characterization of phytoconstituents of the hydro-ethanolic extract of Syzygium cumini seed (SCE). Further, this report covers the molecular docking findings of the bioactive constituents on the sulfonylurea receptor 1 (SUR1). Methods: A glucose uptake assay of SCE was used to estimate the glucose uptake from the cell lysates and the cell culture supernatants using insulin as the reference standard. Insulin release activity of SCE from RIN-5F cells was estimated using enzyme-linked immunosorbent assay. The phytoconstituents were isolated by preparative HPLC and characterized by mass spectrometry, nuclear magnetic resonance (NMR) and infrared spectroscopy. The molecular docking of bioactive constituents was carried on repaglinide bound to the SUR1.

Results: In the presence of SCE, the glucose uptake through L6 myoblast cells increased by $19.91 \%$ at $40 \mu \mathrm{g} / \mathrm{mL}$ in comparison with the vehicle control $(P<0.05)$. Moreover, SCE showed 2.8 -fold enhancement of insulin release at $40 \mu \mathrm{g} / \mathrm{mL}$ as compared to the vehicle controls $(P<0.05)$. Gallic and ellagic acids were the key phytoconstituents isolated from SCE. Molecular docking studies revealed that both gallic acid and ellagic acid bind to the repaglinide binding pocket of SUR1.

Conclusion: SCE increases the release of insulin and enhances glucose uptake in vitro, which may contribute to its in vivo anti-diabetic activity. The presence of ellagic acid and gallic acid in SCE may be the cause for enhanced insulin release observed with SCE following binding to SUR1.
\end{abstract}

Implication for health policy/practice/research/medical education:

Syzygium cumini increased the release of insulin and enhanced glucose uptake in vitro. The research is relevant to public health because it focuses on the mechanism of insulin secretion and its relevance for type-2 diabetes treatment.

Please cite this paper as: Asanaliyar M, Nadig P, Bharatam N. In vitro anti-diabetic activity, bioactive constituents, and molecular modeling studies with sulfonylurea receptorl for insulin secretagogue activity of seed extract of Syzygium cumini (L.).

J Herbmed Pharmacol. 2021;10(3):304-312. doi: 10.34172/jhp.2021.35.

\section{Introduction}

Type-2 diabetes mellitus (DM) is diagnosed when there is an elevation of fasting blood glucose (FBG) of more than $126 \mathrm{mg} / \mathrm{dL}$ and postprandial blood glucose (PPBG) of more than $200 \mathrm{mg} / \mathrm{dL}$ (1). The pathophysiological manifestation of type-2 diabetes includes peripheral insulin resistance, impaired regulation of hepatic glucose production, and decline of $\beta$-cell function, eventually leading to $\beta$-cell failure (2). Insulin resistance (IR) is generally defined as a reduced ability of insulin to induce glucose uptake by target tissues such as adipose tissue and the skeletal muscle. 
The current management of type-2 DM involves the reduction of hepatic glucose output with biguanides like metformin, decreasing insulin resistance using thiazolidinediones such as pioglitazone, enhancing insulin secretion with sulphonylureas such as glimepiride or DPP4 inhibitors (sitagliptin) and reducing the dietary absorption with acarbose like alpha-glucosidase inhibitors (3).

The World Health Organization (WHO) expert committee on diabetes has recommended evaluation of the traditional methods of managing this disease because of the high mortality, morbidity, complications, and problems associated with the use of conventional antidiabetic agents (4). Further, medicinal plant extracts, with their multiple phytoconstituents, are known to exert their actions at multiple targets cohesively, leading to synergistic outcomes (5). Therefore, there is an everincreasing interest in validating medicinal plant extracts for type-2 DM as these natural products could prove to be a very good source of new drugs to treat diabetes.

Syzygium cumini also termed Eugenia jambolana is called Jamun in Hindi. Jamun seeds are mentioned in the ayurvedic pharmacopoeia as a medication for diabetes. Taking this lead from Ayurveda, extracts of different parts of this plant have been tested for antidiabetic activities and among these the seeds have shown more promise (6).

Alcoholic extract of Syzygium cumini seed (SCE) showed significant anti-diabetic activity at $100 \mathrm{mg} / \mathrm{kg}$ in alloxan-induced diabetic rats (7). The HPLC profiling of $70 \%$ methanol extracts (ME) was found to be enriched in phenolic compounds, namely ellagic acid and gallic acid. This extract has been demonstrated to have significant antioxidant activity in vitro (8). A double-blind, randomized controlled trial of $10 \mathrm{~g} /$ day SCE powder in type-2 DM patients showed a beneficial effect in reducing blood sugar, blood pressure, and in improving the quality of life (9).

Our in vivo experiments with SCE at doses of 100 and $200 \mathrm{mg} / \mathrm{kg}$ body weight in Wistar albino rats demonstrated a significant improvement in anti-diabetic activity in highfat diet and low dose streptozotocin-induced experimental type-2 diabetes model. There was a reduction in FBG and increase in serum insulin. Reduction in insulin resistance as measured by homeostasis model of assessment for insulin resistance (HOMA-IR) and an improved pancreatic beta-cell function measured by homeostasis model of assessment for beta-cell function (HOMA-B) have been observed before, with a more pronounced effect on beta-cell function (unpublished data). Further, SCE at $200 \mathrm{mg} / \mathrm{kg}$ has also shown an increased expression of glucose transporter 4 (GLUT-4) gene in the skeletal muscle in high-fat diet and low dose streptozotocin model (unpublished data), indicating an improvement in insulin resistance profile.

The present study was, therefore, conducted with the aim of exploring the mechanism of action of SCE on pancreatic beta cells and skeletal muscle. The specific objectives were to observe whether it enhances the insulin release from RIN 5F cells ( $5 \mathrm{~F}$ clone of rat insulinoma cells) and the glucose uptake in skeletal muscle using immortalized rat L6 myoblast cells in vitro. An attempt was also made to isolate and characterize some of the phytochemical constituents from SCE, run molecular docking on sulfonylurea receptor 1 (SUR1)/pancreatic ATP-sensitive $\mathrm{K}^{+}$channel structure studies in order to explain the observed in vitro anti-diabetic effects.

\section{Materials and Methods}

\section{Chemicals}

All the chemicals and reagents used in this study were purchased from Sigma-Aldrich Inc (St Louis, MO, USA). L6 cells and RIN-5F cells were procured from ATCC [CL173], a- MEM \& Insulin from Sigma, Horse serum from Invitrogen, DMEM from GIBCO, BSA, Insulin from GEAmersham, UK.

\section{Plant material}

The standardized hydro-ethanolic extract of SCE was procured from Natural remedies, Bangalore (Batch number; FSCEX/2015090001). The extract was characterized and standardized by the manufacturer to contain polyphenols ( $29.4 \%$ by spectrophotometry) and ellagic acid (2.8\% by high-performance liquid chromatography). The levels of heavy metal content, microbial counts, aflatoxin, and residual solvents were in compliance with British Pharmacopeia/United States Pharmacopoeia standards (data on file).

Determination of cell viability for in vitro studies

It is essential to determine the effects of the extract on cell viability at various concentrations so as to choose appropriate concentrations to evaluate the anti-diabetic activity in vitro. Hence, the cell viability for both RIN-5F and L6 cells was determined using 3-(4, 5-dimethylthiazol2-yl)-2, 5-diphenyltetrazolium bromide (MTT) reduction assay (10,11). The anti-diabetic activity assay was conducted at concentrations of $10,20,40$, and $80 \mu \mathrm{g} / \mathrm{mL}$.

Glucose uptake assay of SCE on L6 myoblast cells Glucose uptake activity of SCE was determined in differentiated L6 myoblast cells as per Takigawa-Imamura et al, and Syama et al $(12,13)$. The cells were treated with non-toxic concentrations of SCE extract $(10,20$, and $40 \mu \mathrm{g} / \mathrm{mL}$ ) and insulin (positive control) at $100 \mathrm{nM}$. $\mathrm{D}$-glucose solution was added to each well and incubated at $37^{\circ} \mathrm{C}$ for 30 minutes. Then, the culture supernatant and all the treatment substances were aspirated, and the cells were washed thrice with ice-cold Krebs-Ringer-Phosphate buffer solution before using them to estimate glucose uptake. The cells were lysed with $0.1 \mathrm{M} \mathrm{NaOH}$ solution 
and an aliquot of the lysates used to measure the cellular glucose levels using a glucose assay kit. Every assay was performed in duplicate and on three independent occasions. The enhancement (\%) of glucose uptake over controls was then calculated.

Insulin secretion activity of SCE in RIN-5Fcells For determining the insulin secretion activity of SCE in RIN-5Fcells a previous method was followed (14). RIN$5 \mathrm{~F}$ cells, which are clonal cells derived from rat pancreatic beta cells, were used to evaluate insulin secretion activity in this study. Varying concentrations of SCE $(1.25,2.5,5$, 10,20 , and $40 \mu \mathrm{g} / \mathrm{mL}$ ) and standard (1mM glucose) were tested. The amount of secreted insulin was expressed as $\mu \mathrm{U} / 1 \times 10^{6}$ cells. Fold stimulation was estimated as insulin secretion after various treatments compared to that with $1 \mathrm{mM}$ glucose treated cells, which was used for normalization.

\section{Isolation and characterization of bioactive constituents of SCE}

Seven g of SCE powder was extracted with ethanol, filtered, and then concentrated to get the crude product of $3 \mathrm{~g}$. The crude product was dissolved in a $20 \mathrm{~mL}$ mixture of water: tetrahydrofuran in 1:1 ratio and purified by preparative high-performance liquid chromatography (HPLC, Agilent 1260 Infinity). The conditions for HPLC were as follows: Mobile phase, A: $0.1 \%$ Formic acid in water, B: Acetonitrile; Flow rate: $20.0 \mathrm{~mL} / \mathrm{min}$; Column: Sunfire C18 $(50 \times 50 \mathrm{~mm}), 10 \mu \mathrm{m}$.

The above preparative HPLC separation yielded $300 \mathrm{mg}$ of compound-1 with liquid chromatography -Mass spectrometry (LCMS) purity $71 \%$ and $350 \mathrm{mg}$ of compound-2 with LCMS purity $76 \%$ as major bioactive constituents. The isolated compound-1 and compound- 2 were further purified to get $50 \mathrm{mg}$ of pure compound-1 with HPLC purity $>95 \%$ and $45 \mathrm{mg}$ of compound- 2 with HPLC purity $>95 \%$. The conditions used for the second purification were as follows: Mobile phase, A: $0.1 \%$ Formic acid in water, B: Acetonitrile; Flow rate: $20.0 \mathrm{~mL} /$ min; Column: X-bridge C8 $(19 \times 150 \mathrm{~mm}), 5 \mu \mathrm{m}$. Diode array detector in the UV detection range of 210-400 nm was used for identification in both the steps.

The purity of isolated compound-1 and compound-2 was further confirmed by HPLC (Method info: A: $0.1 \%$ formic acid in $\mathrm{H} 2 \mathrm{O}$, B: Methanol, Flow rate: $1.0 \mathrm{~mL} / \mathrm{min}$. Column: X-bridge C8 (50X4.6) mm, $3.5 \mu \mathrm{m})$. Structural characterization of compound-1 and compound-2 was done using Fourier transformer infrared (FT-IR) spectroscopy, mass spectrometry, and nuclear magnetic resonance (NMR) spectroscopy.

Molecular docking methods Ligand and protein preparation for docking simulations The 2D structures of gallic acid and ellagic acid were prepared using the Ligprep module in the Schrodinger suite (15). Ligprep accepts molecules in 2D format and converts them to 3D. The Epik sub-module of Ligprep was used to generate tautomers and possible ionization states for each molecule. Each ligand was manually inspected to ensure that the correct tautomer state and ionization state at physiologically relevant $\mathrm{pH}$ (7.4). Output structures from Ligprep were considered as input for multiple conformations generation using the MacroModel-MTLM (mixed torsional/low-mode) method. MacroModelMTLM combines a Monte Carlo method of exploring torsional space (that efficiently locates widely separated minima on a potential energy surface) with a low-mode conformational search method that searches along energetically "soft" degrees of freedom. The OPLS2005 force field was used and energy minimization was performed for 500 steps using the TNCG method. The energy window for acceptable structures was set to $21 \mathrm{~kJ} /$ mol (default value). Conformations of the same molecule within $0.5 \AA$ RMSD were culled. A maximum of 500 steps was allowed for Monte Carlo sampling and a maximum of 50 steps was allowed for low mode searching. A maximum of 20 conformers per molecule was accepted.

Repaglinide bound to the SUR1/pancreatic ATPsensitive $\mathrm{K}^{+}$channel structure (PDB ID: 6JB3) was processed initially using the Protein Preparation Wizard in the Maestro suite from Schrodinger to add the correct protonation state and bond orders to hetero atoms of protein residues, water molecules, and the bound ligands. The digitonin molecule bound within $6 \AA$ of the bound repaglinide was removed while docking simulations since it is a detergent used during protein purification and not physiologically relevant (16).

\section{Molecular docking}

The Glide v8.5 (17) molecular docking module (Schrodinger, 2019) in SP-mode was used to generate unbiased binding modes for gallic acid and ellagic acid. The bound repaglinide molecule was considered to assign substrate-binding pocket. Th2 processed complex was used to generate the pre-computed docking grid. The docking protocol started with the ligand's systematic conformational expansion followed by placement in the receptor site. Minimization of the ligand in the receptor's field was then carried out using the OPLS-AA force field with the default distance-dependent dielectric. The lowest energy poses were then subjected to a Monte Carlo procedure that sampled nearby torsional minima. Poses were ranked using GlideScore, a modified version of the ChemScore function that includes terms for steric clashes and buried polar groups. The default Van der Waal's scaling was used (1.0 for the receptor and 0.8 for the ligand). The resultant dock poses were visualized manually in PyMOL software. 


\section{Results}

Effect of SCE on cell viability

All the concentrations of SCE exhibited less than 20\% toxicity up to a concentration of $100 \mu \mathrm{g} / \mathrm{mL}$, and thus, a maximum concentration of $40 \mu \mathrm{g} / \mathrm{mL}$ was used for glucose uptake and insulin release studies.

Effect of SCE on glucose uptake in L6 myoblast cells As shown in Figure 1, insulin at 100nM showed 55.6\% glucose uptake while SCE showed a concentration dependent increase in the glucose uptake with a maximum activity of $19.91 \%$ uptake at $40 \mu \mathrm{g} / \mathrm{mL}$ compared to the vehicle control (7.60\%).

Effect of SCE on insulin secretion from RIN 5F cells Figure 2 represents the effect of SCE on insulin secretory

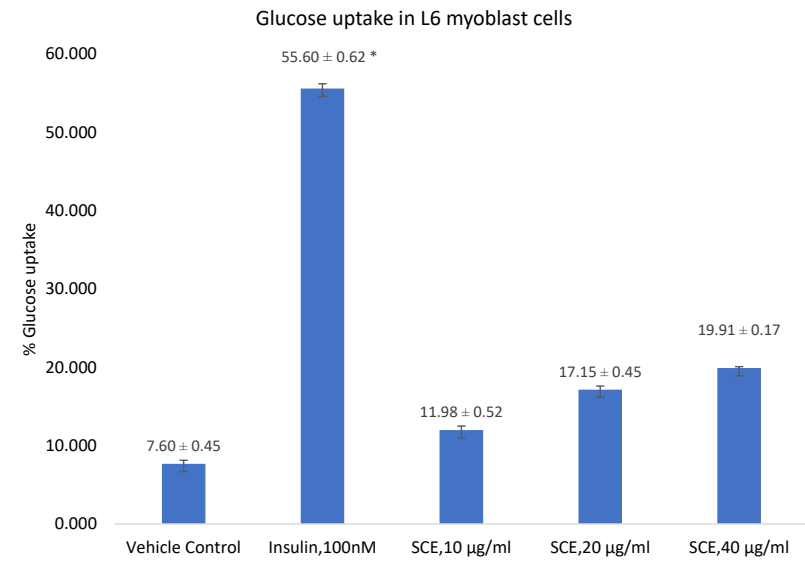

Figure 1. Effect of Syzygium cumini (SCE) on glucose uptake in isolated L6 myoblast cells. The values indicate mean percent glucose uptake \pm standard deviation compared to the insulin (positive control). Each value is a mean of the tests done in triplicates. ${ }^{*} P<0.05$ compared to normal control.

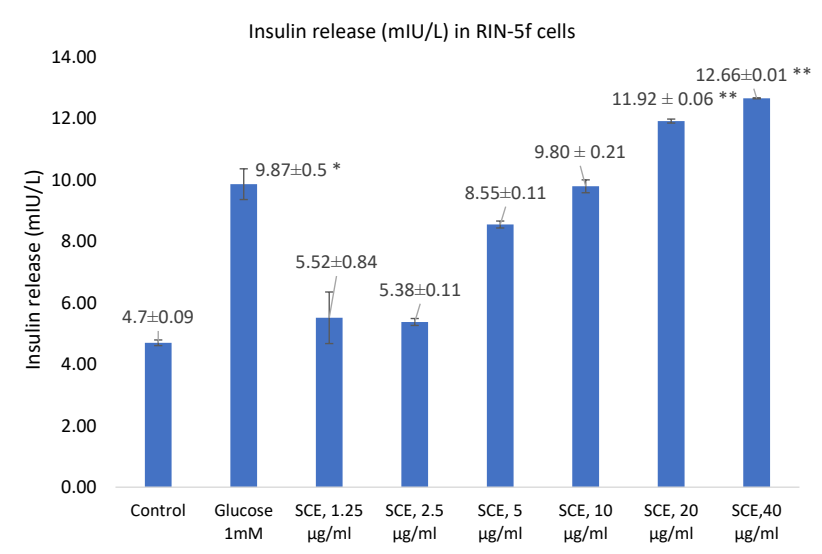

Figure 2. Effect of Syzygium cumini extract (SCE) on insulin release ( $\mathrm{mIU} / \mathrm{L}$ ) in isolated RIN 5F cells. The values indicate mean insulin release Istandard deviation compared to the positive control. Each value is a mean of tests done in triplicates. ${ }^{*} P<0.05$ compared to normal control; ${ }^{* *} P<0.05$ compared to positive control

activity in RIN 5F cells. The standard (positive) control used in this study was $1 \mathrm{mM}$ glucose. The insulin release with glucose was found to be $9.87 \mathrm{mIU} / \mathrm{L}$, which was a 2.0 -fold increase while with $40 \mu \mathrm{g} / \mathrm{mL}$ of SCE, it was 12.87 $\mathrm{mIU} / \mathrm{L}$, which was a 2.8 -fold increase compared to the vehicle control $(4.7 \mathrm{mIU} / \mathrm{L}, P<0.05)$.

Isolation and structural assignment of bioactive constituents of SCE

Mass spectrometry analysis of compound-1 (Figure 3) showed $\mathrm{m} / \mathrm{z}$ ionization peak value of 169.1 [M-H]. The $\mathrm{m} / \mathrm{z}$ value 169.1 corresponded to the known phytoconstituent gallic acid present in various extracts of S. cumini, and hence compound-1 was considered most probably to be gallic acid. Further ${ }^{1} \mathrm{H},{ }^{13} \mathrm{C}$ NMR (Figure 4) and FT-IR recording of compound- 1 and analysis of recorded NMR<smiles>O=C(O)c1cc(O)c(O)c(O)c1</smiles>

Compound-1 (Gallic acid)

(b)
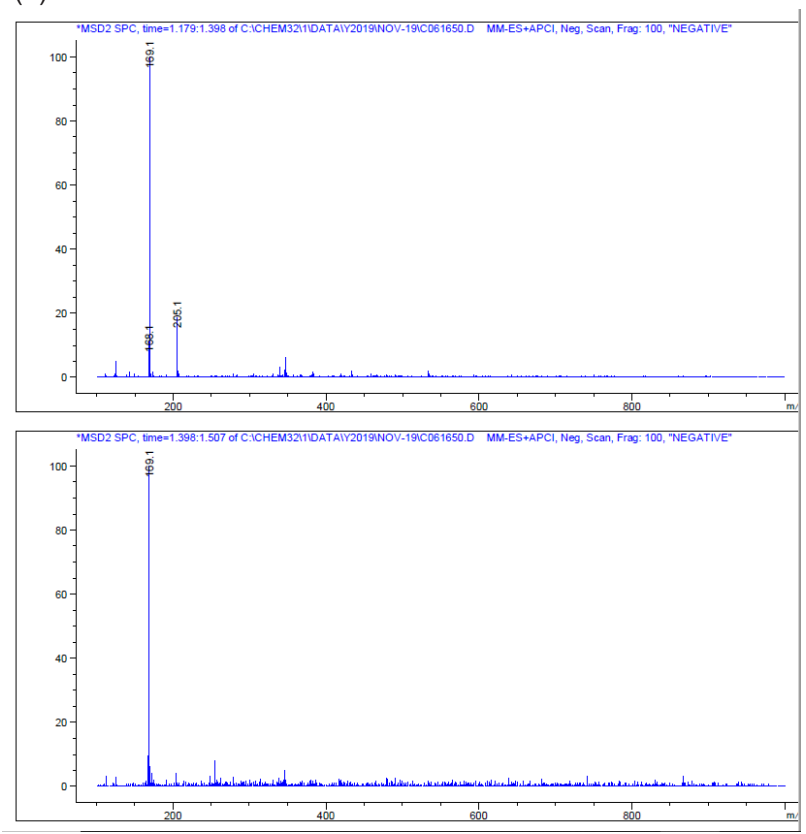

Figure 3. (a) Structures of compound-1 (gallic acid) and compound-2 (ellagic acid). (b) Mass spectrometry profile of isolated compound-1 (Gallic acid).

LCMS = Calculated for $\mathrm{C}_{6} \mathrm{H}_{7} \mathrm{O}_{5}$ 170.02, Observed $=169.1(\mathrm{M}-1)$ : NMR ${ }^{1} \mathrm{H}(400 \mathrm{MHz}$, Acetone-d6) $\delta$ ppm: 3.3 (sa, 4H, OH-4), 7.14 (s, 2H, H-2, $\mathrm{H}-6$ ), 8.42 (sa, 2H, OH-3, OH-5); NMR ${ }^{13} \mathrm{C}(400 \mathrm{MHz}$, Acetone-d 6) $\delta$ ppm: 109.21 (C2, C6), 121.18 (C1), 137.66 (C4), 145.18 (C3, C5), 166.94 (COOH); FT-IR (cm-1): 3404.02, 3276.25, 1668.01,1608.44, 1423.88, $1315.86,1263.45,1191.91,1024.1,723.19,567.09 \mathrm{~cm}^{-1}$ 
and IR spectrum of compound-1 suggested the presence of characteristic peak corresponding to gallic acid structure. The compound-1 recorded NMR spectroscopic data matched with that of the reference standard gallic acid (20).

Similarly, the mass spectrometry (Figure 5) analysis of compound-2 showed $\mathrm{m} / \mathrm{z}$ ionization value 302.8 (Mass value). The $\mathrm{m} / \mathrm{z}$ value 302.8 corresponded to the known bioactive constituent ellagic acid present in various extracts of S. cumini, and hence compound-2 was considered to be probably ellagic acid. Further ${ }^{1} \mathrm{H}$ NMR (Figure 6) and FT-IR recording of compound-2 and analysis of corresponding spectrums of compound- 2 elucidated the structure of compound-2 to be ellagic acid. The recorded analytical data of compound- 2 matched with that of the reference standard ellagic acid (19).

\section{Molecular docking}

As the repaglinide bound SUR1 complex structure, which we considered for docking studies is from Mesocricetus

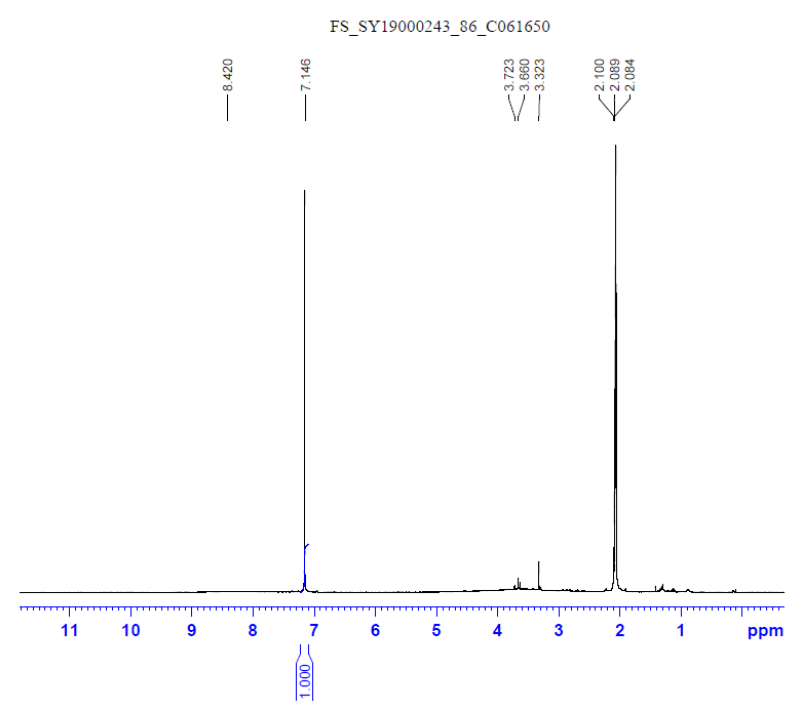

Figure 4. ${ }^{1} \mathrm{H}$ NMR spectrum of isolated compound-1 (gallic acid) auratus and not from human, we investigated the conservation of SUR1 binding pockets between $M$. auratus and human sources. Pairwise sequence comparison studies by protein blast method revealed that the overall sequence identity to be $>95 \%$ between the two sequences. Next, when the conservation of repaglinide binding pocket residues was checked, it became obvious that all the binding pocket residues were conserved between $\mathrm{M}$. auratus and human (Table 1) and negated the generation of human SUR1 homology model for binding mode predictions.

Initially, we examined the repaglinide binding pocket to understand key hydrogen bond and hydrophobic interactions. Repaglinide forms several hydrophobic interactions with Y377, I381, W430, F433, L434, M441, L592, and V596 of SUR1 (Figure 7A). A couple of key hydrogen bond interactions was observed with N1245 and R1246 side chains. To optimize docking protocol and cross-check binding mode prediction ability of molecular docking, we approached re-docking (20) of repaglinide. Comparison of molecular docking predicted binding mode with experimental binding mode (Figure 7A) revealed that Glide docking reproduced known binding mode successfully. We have predicted binding mode for another reference compound (glibenclamide) that shows hydrogen bonding and hydrophobic interactions analogous to repaglinide (Figure $7 \mathrm{~B}$ ). The sulphonyl group forms hydrogen bond interactions with N1245 and R1246 side chains whereas aliphatic and aromatic rings of the molecule form hydrophobic interactions. The Glide docking scores are comparable for the two reference compounds (Table 2).

Analyses of gallic acid and ellagic acid binding modes revealed that these compounds interact in a similar mode like that of the reference compounds (repaglinide \& glibenclamide). The carboxyl group of gallic acid forms hydrogen bond interaction with R1246 and similar to repaglinide, whereas hydroxyl group of the $3^{\text {rd }}$ and $4^{\text {th }}$ position forms hydrogen bond interaction with F433 main

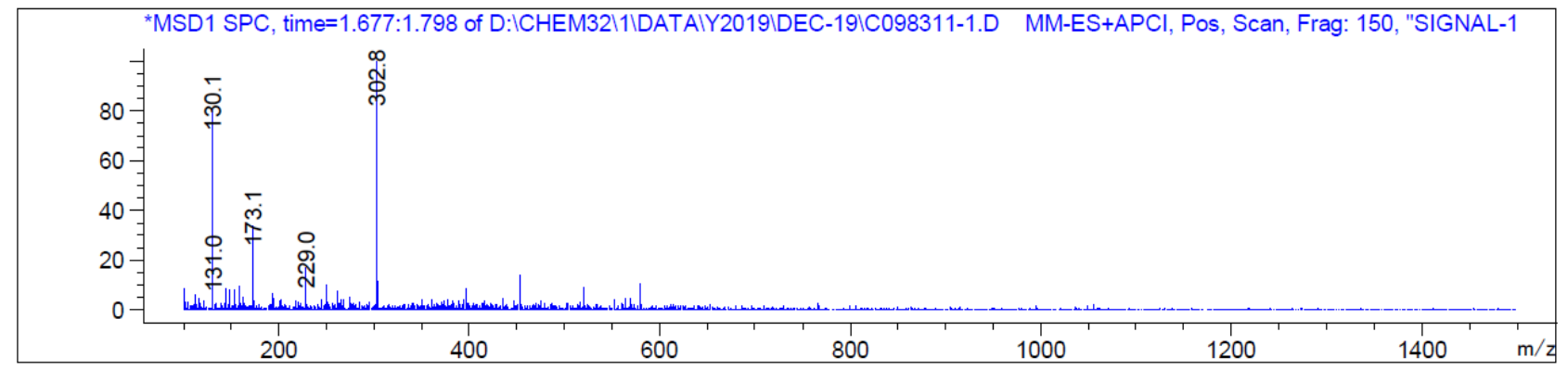

Figure 5. Mass spectrometry profile of isolated compound 2 (ellagic acid). LCMS = Calculated for $\mathrm{C}_{14} \mathrm{H}_{6} \mathrm{O}_{8} 302.02$, Observed $=302.18\left(\mathrm{M}^{+}\right):(\mathrm{DMSO}-\mathrm{d} 6)$ ppm: $\delta 7.46(\mathrm{~s}, 2 \mathrm{H}, \mathrm{ArH}), 10.60-10.82(\mathrm{~m}, 4 \mathrm{H},-\mathrm{OH}): \mathrm{FT}-\mathrm{IR}\left(\mathrm{cm}{ }^{-1}\right): 2800-3700$, $1725,1669,1588,1190,1052,751 \mathrm{~cm}^{-1}$. 


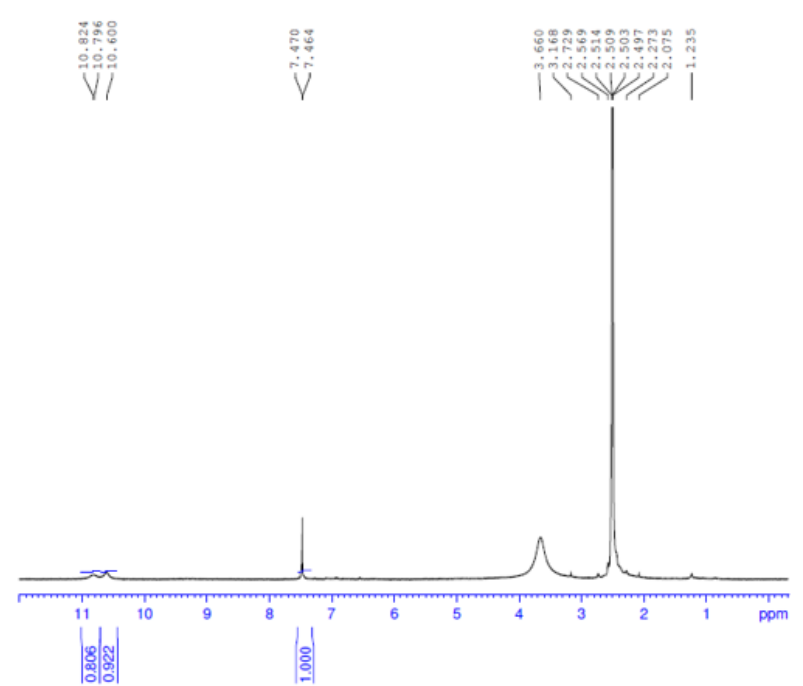

Figure 6. ${ }^{1} \mathrm{H}$ NMR spectrum of isolated compound-2 (Ellagic acid)

chain carbonyl group (Figure 7C). The phenyl ring forms hydrophobic interactions with side chains of I381, W430, F433, L434 and Y377 residues. Ellagic acid, a dimeric form of gallic acid also forms hydrogen bond interaction with R1246 and demonstrated hydrogen bond interactions with the F433 main chain carbonyl group (Figure 7D). Ellagic acid shows several stacking and hydrophobic interactions with hydrophobic residues (I381, W430, F433, L434 and Y377).

\section{Discussion}

Herbal extracts are safer compared to the chemical drugs as claimed in the Indian and Chinese traditional medicine. However, when extracted with different solvents, their properties are likely to change. Hence, they must be validated thoroughly for their efficacy and safety before recommending them for human use. We conducted our experiments towards this objective and found that the hydro-ethanolic extract of S. cumini showed significant anti-diabetic effects in vivo in experimental diabetes. It was also nontoxic at 10 times the anti-diabetic dose in acute toxicity studies in vivo conducted as per Organization for Economic Co-operation and Development (OECD) guidelines (data on file).

The study demonstrated that the hydro-ethanolic seed extract of S. cumini enhanced the glucose uptake and increased the insulin secretion in the in vitro systems and was not cytotoxic up to a concentration of $100 \mu \mathrm{g} / \mathrm{mL}$. The extract showed the presence of ellagic acid and gallic acid.

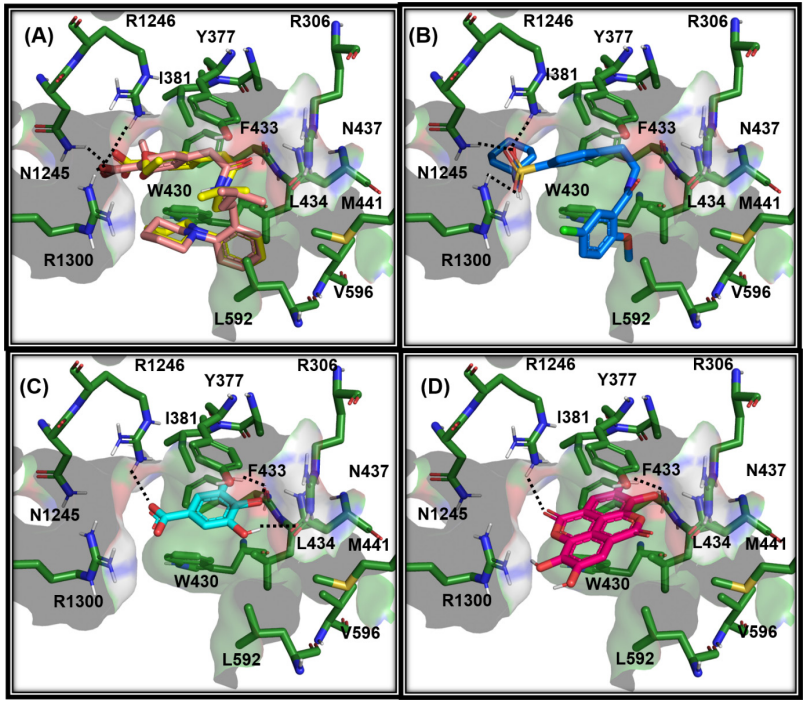

Figure 7. Molecular docking predicted binding modes of repaglinide (A), glibenclamide (B), gallic acid (C), and ellagic acid (D). Repaglinide experimental binding mode shown as yellow sticks in panel $(A)$ and predicted dock pose depicted as pink sticks and superposed for comparison. Key binding pocket residues shown as green sticks in all panels and inter-molecular hydrogen bond interactions are depicted as broken lines. Glibenclamide, gallic acid, and ellagic acid dock poses shown as blue, cyan, and magenta sticks in respective panels.

Our study appears to be the first one to demonstrate the in vitro anti-diabetic effects of hydro-ethanolic seed extract of S. cumini.

The glucose uptake activity of SCE was evaluated in isolated L6 myoblast cells. Isolated L6 myotubules have proven to be a suitable model for in vitro glucose uptake. Yonemitsu et al have demonstrated increased glucose uptake with thiazolidinediones and attributed this to an increase in GLUT-4 expression in these cells (21). SCE showed a concentration-dependent increase in glucose uptake activity, and the maximum activity was observed at $40 \mu \mathrm{g} / \mathrm{mL}$ with $19.91 \%$ uptake. Earlier studies on methanolic and ethanolic seed extracts of S. cumini have demonstrated a similar increase in the glucose uptake activity in L6 cell lines through GLUT4 translocation $(11,22)$. The observed glucose uptake activity was attributed to the presence of polyphenols present in these extracts. Gallic and ellagic acid, plant-based polyphenolic compounds present in S. cumini, have shown to increase GLUT4 translocation and glucose uptake activity in 3T3-L1 cells (cell line derived from mouse used for biological research on adipose tissue). Gallic acid induces glucose uptake in a phosphatidylinositol-3kinase (PI3K) dependent manner through activation of

Table 1. Sulfonylurea receptor 1 (SUR1) binding pocket conservation between Mesocricetus auratus and human

\begin{tabular}{llllllllllll}
\hline Mesocricetus auratus & Y377 & I381 & W430 & F433 & L434 & M441 & L592 & V596 & N1245 & R1246 & R1300 \\
\hline Human & Y377 & I381 & W430 & F433 & L434 & M441 & L592 & V596 & N1244 & R1245 & R1299 \\
\hline
\end{tabular}

Y-Tyrosine; I-Isoleucine; W-Tryptophan; F-Phenylalanine; L-Leucine; M- Methionine; V-Valine; N- Asparagine; R-Arginine. 
Table 2. Glide molecular docking scores of four compounds with sulfonylurea receptor 1 (SUR1)

\begin{tabular}{lc}
\hline Compound & Glide dock score (kcal/Mol) \\
\hline Repaglinide & -6.9 \\
Glibenclamide & -6.8 \\
Gallic acid & -5.3 \\
Ellagic acid & -5.6 \\
\hline
\end{tabular}

protein kinase $\mathrm{C}$ zeta/lambda $(\mathrm{PKC} \zeta / \lambda)$ as a downstream effector of PI3K activation (23), whereas the ellagic acid is reported to stimulate glucose uptake through adenosine monophosphate-activated protein kinase mediated pathway (24). In this study, we have reported the isolation of gallic and ellagic acid from SCE, and thus the glucose uptake activity of the extract could probably be due to these bioactive constituents.

Stimulus secretion coupling events promote the increase of adenosine triphosphate/adenosine diphosphate (ATP/ $\mathrm{ADP})$ ratio with consequent closure of ATP-sensitive $\mathrm{K}^{+}$channels ( $\left.\mathrm{K}^{+} \mathrm{ATP}\right), \beta$-cell membrane depolarization and $\mathrm{Ca}^{2+}$ influx that trigger insulin-containing granules exocytosis (25).

Repaglinide is a known short-acting insulin secretagogue widely prescribed for the treatment of type- 2 diabetes and it boosts insulin secretion through inhibition of pancreatic ATP-sensitive potassium channel (KATP) via binding to the SUR1 subunit (16).

The present study demonstrated the insulin secretagogue activity of SCE in rat pancreatic beta cells (RIN-5F) when treated with 1.25 to $40 \mu \mathrm{g} / \mathrm{mL}$ of the extract and it improved insulin secretion activity in a concentration-dependent manner with the maximal response (12.66 mIU/L) observed at $40 \mu \mathrm{g} / \mathrm{mL}$. SCE produced a significantly higher insulin secretion than $1 \mathrm{mM}$ glucose that gave a response of $9.87 \mathrm{mIU} / \mathrm{L}$ and showed a dose-dependent increase in insulin release. This suggests that SCE may probably act as insulin secretagogue and may circumvent $\beta$-cell failure associated type- 2 diabetic condition in patients. The insulin secretagogue effect of SCE can be attributed to the presence of gallic acid and ellagic acid as both of them possess insulin secretion activity in RINm5F $\beta$-cells and on isolated mice islets $(14,26)$.

It is possible that both gallic and ellagic acids present in SCE exert insulin secretagogue activity through inhibition of pancreatic ATP-sensitive potassium channel (KATP) by binding to the SUR1 subunit which is the reported mechanism of action for molecules like glibenclamide and repaglinide.

A preparative HPLC directed isolation and characterization by MS/MS analysis was done to identify the probable phytoconstituents present in SCE. The MS spectral data for compounds $1 \& 2$ were compared with the published data to assign the structure of the isolated compound. Compound-1 was identified as gallic acid, as it showed major ionization peak (M-1) at $\mathrm{m} / \mathrm{z}$ value of 169.1, which corresponds to gallic acid molecular weight. Further analysis of ${ }^{1} \mathrm{H}$ NMR, ${ }^{13} \mathrm{C}$ NMR and FTIR compound-1 suggested that the isolated compound-1 from SCE extract could be gallic acid and it matches with that of reference gallic acid (18).

In a similar way, compounds- 2 showed major ionization peak $\left[\mathrm{M}^{+}\right]$at $\mathrm{m} / \mathrm{z}$ value of 302.8 , which corresponds to ellagic acid molecular weight. Further, the analysis of MS, ${ }^{1} \mathrm{HNMR}$, and FT-IR of compound-2 elucidated the structure as ellagic acid and the recorded analytical data matched with that of the reference standard of ellagic acid (19). To the best of our knowledge, this is the first time that gallic and ellagic acid have been reported in the hydro-ethanolic extract of SCE seeds, which may be partly responsible for glucose uptake and insulin secretion activity observed with the in vitro assays.

In order to link the excellent insulin secretagogue activity observed for SCE through insulin release assay to molecular-level understanding, we carried out molecular docking studies for gallic and ellagic acid using repaglinide bound to the SUR1/pancreatic ATP-sensitive $\mathrm{K}^{+}$channel structure (PDB ID: 6JB3).

Though we have observed highly identical predicted binding mode compared with experimental mode, the ethoxy group of repaglinide was predicted to be inside a hydrophobic groove formed by I381, W430, and F433 side chains. The ethoxy group is solvent exposed in the experimental structure (Figure 7A). We believe that the ethoxy group preferentially occupies the hydrophobic groove or both orientations are possible in experimental conditions. But we observed only one orientation, which might be due to force field preference towards hydrophobic interactions and scoring weightage.

All the four compounds (repaglinide, glibenclamide, gallic and ellagic acids) considered in docking studies have shown hydrogen bond interaction with R1246, whereas reference compounds (repaglinide, glibenclamide) demonstrated extra hydrogen bond interactions with side chains of N1245 and R1300. Point mutations studies revealed that $\mathrm{R} 1246$ is a key residue for repaglinide as well as glibenclamide binding (18). In our docking studies, both gallic acid and ellagic acid were predicted to interact with this residue. These two compounds also exhibited 2-3 additional hydrogen bond interactions with binding pocket residues due to the presence of hydroxyl groups. The dock scores of these two compounds are slightly lower compared to reference compounds, which is expected due to the size as well as the lack of flexibility. The molecular docking studies explain that both gallic and ellagic acid bind to the same site as that of repaglinide site in SUR1/ pancreatic ATP-sensitive $\mathrm{K}^{+}$channel structure and make similar molecular interactions in the active site. Hence, 
the insulin secretion activity observed for SCE extract may be due to the binding of gallic and ellagic acids to SUR1 and thus inhibition of pancreatic ATP-sensitive $\mathrm{K}^{+}$ channel.

The study thus validated the traditional use of S. cumini for its anti-diabetic properties. There was an enhanced glucose uptake by the L6 myoblasts and insulin release by the RIN-5F cells. Further, ellagic acid and gallic acid appear to be important phytoconstituents of SCE, which may be responsible for the observed effects. Molecular docking studies using SUR1/pancreatic ATP-sensitive $\mathrm{K}^{+}$channel structure prove that the insulin secretagogue activity could be due to inhibition of this channel through binding of gallic and ellagic acid to SUR1 subunit. We need to extend such studies with other phytoconstituents. Further exploration also needs to be carried out to identify the exact intracellular molecular mechanisms of action.

\section{Acknowledgement}

The authors are thankful to the management, Principal for providing the necessary infrastructure and all the faculty members of the department of Pharmacology for the support in conducting the experiments.

\section{Authors' contributions}

MA conducted the work, analysed the results and drafted the manuscript. PD conceived the idea, research hypothesis and the protocol for the experiments, contributed to the development and edit of the manuscript. NB conducted the molecular docking studies and contributed to the manuscript writing.

\section{Conflict of interests}

The authors declare no conflict of interest.

\section{Ethical considerations}

Ethical issues of plagiarism, misconduct, data fabrication, falsification, double publication or redundancy have been completely observed by the authors. The study was approved by Institutional Animal Ethics committee (IAEC) of Vydehi Institute of Medical Science and Research Centre (VIMS\&RC), Bangalore, India (VIMS/ IAEC/2016/01).

\section{Funding/Support}

None.

\section{References}

1. Matthaei S, Stumvoll $M$, Kellerer $M$, Häring HU. Pathophysiology and pharmacological treatment of insulin resistance. Endocr Rev. 2000;21(6):585-618. doi: 10.1210/ edrv.21.6.0413.

2. Mahler RJ, Adler ML. Clinical review 102: type 2 diabetes mellitus: update on diagnosis, pathophysiology, and treatment. J Clin Endocrinol Metab. 1999;84(4):1165-71.

\section{doi: $\quad$ 10.1210/jcem.84.4.5612.}

3. Chaudhury A, Duvoor C, Reddy Dendi VS, Kraleti S, Chada A, Ravilla R, et al. Clinical review of antidiabetic drugs: implications for type 2 diabetes mellitus management. Front Endocrinol (Lausanne). 2017;8:6. doi: 10.3389/ fendo.2017.00006.

4. Crandall JP, Knowler WC, Kahn SE, Marrero D, Florez JC, Bray GA, et al. The prevention of type 2 diabetes. Nat Clin Pract Endocrinol Metab. 2008;4(7):382-93. doi: 10.1038/ ncpendmet0843.

5. Zhou X, Seto SW, Chang D, Kiat H, Razmovski-Naumovski V, Chan K, et al. Synergistic effects of Chinese herbal medicine: a comprehensive review of methodology and current research. Front Pharmacol. 2016;7:201. doi: 10.3389/fphar.2016.00201.

6. Asanaliar M, Nadig P. Syzygium cumini (jamun) therapeutic potential: a comprehensive review on phytochemical constituents and emphasis on its pharmacological actions related to diabetic intervention. Int J Basic Clin Pharmacol. 2020;9(2):363-370. doi: 10.18203/2319-2003. ijbcp20200192.

7. Prince PS, Kamalakkannan N, Menon VP. Antidiabetic and antihyperlipidaemic effect of alcoholic Syzigium cumini seeds in alloxan induced diabetic albino rats. J Ethnopharmacol. 2004;91(2-3):209-13. doi: 10.1016/j. jep.2003.11.001.

8. Priya SH, Prakasan N, Purushothaman J. Antioxidant activity, phenolic-flavonoid content and high-performance liquid chromatography profiling of three different variants of Syzygium cumini seeds: a comparative study. J Intercult Ethnopharmacol. 2017;6(1):107-14. doi: 10.5455/ jice. 20161229055555.

9. Sidana S, Singh VB, Meena BL, Beniwal S, Singh K, Kumar $\mathrm{D}$, et al. Effect of Syzygium cumini (jamun) seed powder on glycemic control: a double-blind randomized controlled trial. J Med Soc. 2017;31(3):185-9. doi: 10.4103/jms. jms_62_16.

10. Crouch SP, Kozlowski R, Slater KJ, Fletcher J. The use of ATP bioluminescence as a measure of cell proliferation and cytotoxicity. J Immunol Methods. 1993;160(1):81-8. doi: 10.1016/0022-1759(93)90011-u.

11. Gonzalez RJ, Tarloff JB. Evaluation of hepatic subcellular fractions for Alamar blue and MTT reductase activity. Toxicol In Vitro. 2001;15(3):257-9. doi: 10.1016/s08872333(01)00014-5.

12. Takigawa-Imamura H, Sekine T, Murata M, Takayama K, Nakazawa K, Nakagawa J. Stimulation of glucose uptake in muscle cells by prolonged treatment with scriptide, a histone deacetylase inhibitor. Biosci Biotechnol Biochem. 2003;67(7):1499-506. doi: 10.1271/bbb.67.1499.

13. Syama HP, Arun KB, Sinumol G, Dhanya R, Suseela Anusree S, Nisha P, et al. Syzygium cumini seed exhibits antidiabetic potential via multiple pathways involving inhibition of a-glucosidase, DPP-IV, glycation, and ameliorating glucose uptake in L6 cell lines. J Food Process Preserv. 2018;42(2):e13464. doi: 10.1111/jfpp.13464.

14. Sameermahmood Z, Raji L, Saravanan T, Vaidya A, Mohan V, Balasubramanyam M. Gallic acid protects RINm5F betacells from glucolipotoxicity by its antiapoptotic and insulinsecretagogue actions. Phytother Res. 2010;24 Suppl 1:S83- 


\section{4. doi: 10.1002/ptr.2926.}

15. Chen IJ, Foloppe N. Drug-like bioactive structures and conformational coverage with the LigPrep/ConfGen suite: comparison to programs $\mathrm{MOE}$ and catalyst. J Chem Inf Model. 2010;50(5):822-39. doi: 10.1021/ci100026x.

16. Ding D, Wang M, Wu JX, Kang Y, Chen L. The structural basis for the binding of repaglinide to the pancreatic K(ATP) channel. Cell Rep. 2019;27(6):1848-57.e4. doi: 10.1016/j.celrep.2019.04.050.

17. Friesner RA, Banks JL, Murphy RB, Halgren TA, Klicic JJ, Mainz DT, et al. Glide: a new approach for rapid, accurate docking and scoring. 1. Method and assessment of docking accuracy. J Med Chem. 2004;47(7):1739-49. doi: 10.1021/ jm0306430.

18. Hernández-García E, García A, Avalos-Alanís FG, RivasGalindo VM, Delgadillo-Puga C, Camacho-Corona MDR. Nuclear magnetic resonance spectroscopy data of isolated compounds from Acacia farnesiana (L) Willd fruits and two esterified derivatives. Data Brief. 2019;22:255-68. doi: 10.1016/j.dib.2018.12.008.

19. Goriparti S, Harish MN, Sampath S. Ellagic acid--a novel organic electrode material for high capacity lithium ion batteries. Chem Commun (Camb). 2013;49(65):7234-6. doi: $10.1039 / \mathrm{c} 3 \mathrm{cc} 43194 \mathrm{k}$.

20. Bharatham N, Bharatham K, Shelat AA, Bashford D. Ligand binding mode prediction by docking: $\mathrm{mdm} 2 / \mathrm{mdmx}$ inhibitors as a case study. J Chem Inf Model. 2014;54(2):648-
59. doi: $10.1021 / \mathrm{ci} 4004656$.

21. Yonemitsu S, Nishimura H, Shintani M, Inoue R, Yamamoto $\mathrm{Y}$, Masuzaki $\mathrm{H}$, et al. Troglitazone induces GLUT4 translocation in L6 myotubes. Diabetes. 2001;50(5):1093101. doi: 10.2337/diabetes.50.5.1093.

22. Narmatha M, Maneemegalai S. An in vitro study of Syzygium cumini seed extract on glucose uptake activity in L-6 cell lines. J Drug Deliv Ther. 2019;9(4-A):256-9. doi: 10.22270/jddt.v9i4-A.3419.

23. Prasad CN, Anjana T, Banerji A, Gopalakrishnapillai A. Gallic acid induces GLUT4 translocation and glucose uptake activity in 3T3-L1 cells. FEBS Lett. 2010;584(3):5316. doi: 10.1016/j.febslet.2009.11.092.

24. Poulose N, Vishnu Prasad CN, Nidhina Haridas PA, Anilkumar G. Ellagic acid stimulates glucose transport in adipocytes and muscles through AMPK mediated pathway. J Diabetes Metab. 2011;2(7):149. doi: 10.4172/21556156.1000149 .

25. Sweet IR, Cook DL, DeJulio E, Wallen AR, Khalil G, Callis J, et al. Regulation of ATP/ADP in pancreatic islets. Diabetes. 2004;53(2):401-9. doi: 10.2337/diabetes.53.2.401.

26. Fatima N, Hafizur RM, Hameed A, Ahmed S, Nisar M, Kabir N. Ellagic acid in Emblica officinalis exerts antidiabetic activity through the action on $\beta$-cells of pancreas. Eur J Nutr. 2017;56(2):591-601. doi: 10.1007/s00394-0151103-y. 